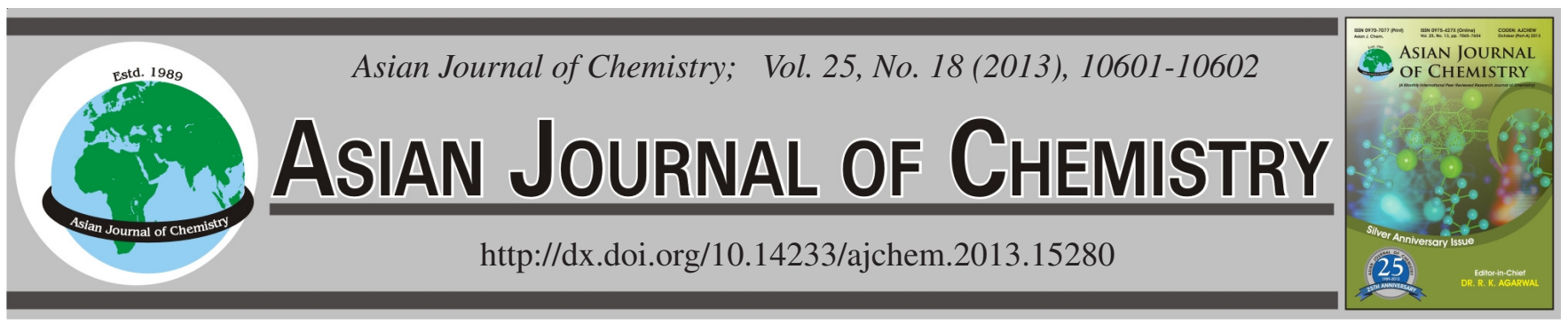

NOTE

\title{
Study on Novel Structure of 4,4'-Bipyridine bis(salicylato)borate Hydrate
}

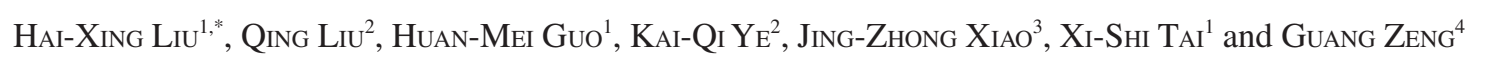

${ }^{1}$ Chemistry \& Chemical and Environmental Engineering College, Weifang University, Weifang 261061, P.R. China

${ }^{2}$ State Key Laboratory of Supramolecular Structure and Materials, College of Chemistry, Jilin University, Changchun 130012, P.R. China

${ }^{3}$ CEMDRX, Department of Physics, University of Coimbra, Coimbra 3004-516, Portugal

${ }^{4}$ State Key Laboratory of Inorganic Synthesis and Preparative Chemistry, College of Chemistry, Jilin University, Changchun 130012, P.R. China

*Corresponding author: E-mail: haixingliu@tom.com

(Received: 5 February 2013;

Accepted: 29 November 2013)

AJC-14457

4,4'-Bipyridine bis(salicylato)borate hydrate with the molecular formula $\left(\mathrm{C}_{10} \mathrm{H}_{10} \mathrm{~N}_{2}\right)\left[\mathrm{B}\left(\mathrm{C}_{7} \mathrm{H}_{4} \mathrm{O}_{3}\right)_{2}\right]_{2}\left(\mathrm{H}_{2} \mathrm{O}\right)_{4}$, is prepared by a hydrothermal reaction and the crystal structure has been determined by means of single-crystal X-ray diffraction. The compound crystallize in monoclinic, system with space group and cell parameters, $C 2 / c, a=24.847 \AA, b=7.4833 \AA, c=21.055 \AA, \alpha=\gamma=90^{\circ}, \beta=112.738^{\circ}, V=3610.7 \AA^{3}$.

The crystal packing is stabilized by $\mathrm{O}-\mathrm{H} \cdots \mathrm{O}$ and $\mathrm{O}-\mathrm{H} \cdots \mathrm{N}$ hydrogen bonding interaction.

Key Words: 4,4'-Bipyridine bis(salicylato)borate, Structure analysis, Hydrogen bonding.

Borate compounds have been the intensive subjects of crystallographic study for the last century. There is a great interest preparing the anhydrous main group or transition metal borate compounds ${ }^{1-3}$. Alkali-metals bis(salicylato)borates have also received the most attention, since the lithium organoborates had been considered as the lithium battery electrolytes ${ }^{4}$. Studies of organic base bis(salicylato)- borates have been less extensive. Piperidinium bis(salicylato)borate is reported in the literature $^{5}$. In this paper, the 4,4'-bipyridine bis (salicylato)borate dihydrate is reported.

All commercially obtained reagent-grade chemicals were used without further purification. A mixture of $\mathrm{SnCl}_{2}(0.1$ mmol, $0.019 \mathrm{~g})$, dilute $\mathrm{HCl}(0.2 \mathrm{~mL}), 4,4$ '-bipyridine $(0.1$ mmol, $0.016 \mathrm{~g})$, salicylic acid $(0.1 \mathrm{mmol}, 0.014 \mathrm{~g})$ and boric acid $(0.1 \mathrm{mmol}, 0.006 \mathrm{~g})$ were added into $20 \mathrm{~mL}$ water with $10 \%$ ethanol and heated for $6 \mathrm{~h}$ at $394 \mathrm{~K}$. The solution was obtained by filtration after cooling the reaction to room temperature. Colourless block single crystals suitable for X-ray measurements were obtained after a few weeks.

The crystal structure of 4,4'-bipyridine bis(salicylato)borate hydrate (Fig. 1) is built up of 4,4'-bipyridine cation, bis(salicylato)borate anion and water molecules. The crystal data and structure refinement is shown in Table-1. Boron atom is coordinated by four $\mathrm{O}$ atoms from two salicylic acid. The distance $\mathrm{d}(\mathrm{B}-\mathrm{O})$ are in the range of 1.441-1.486 $\AA$. The angles of O-B-O are in the range of 106.0-112.8 . The torsion angles of C1-O1-B1-O6, C3-O3-B1O4 and C10-O6-B1-O1 are 99.0,143.8 and 90.9 ${ }^{\circ}$, respectively. Selected bond lengths and bond angles are shown in Table- 2 .

\begin{tabular}{|c|c|}
\hline \multicolumn{2}{|c|}{$\begin{array}{l}\text { TABLE-1 } \\
\text { CRYSTAL DATA AND STRUCTURE } \\
\text { REFINEMENT FOR THE TITLE COMPLEX }\end{array}$} \\
\hline Empirical formula & $\mathrm{C}_{38} \mathrm{H}_{34} \mathrm{~B}_{2} \mathrm{~N}_{2} \mathrm{O}_{16}$ \\
\hline Formula weight & 796.29 \\
\hline Temperature & 293(2) K \\
\hline Wavelength & $0.71073 \AA$ \\
\hline Crystal system, space group & Monoclinic, $\mathrm{C} 2 / \mathrm{c}$ \\
\hline Unit cell dimensions & $\begin{array}{l}\mathrm{a}=24.847(5) \AA \AA \alpha=90^{\circ} \\
\mathrm{b}=7.4833(15) \AA \beta=112.738(2)^{\circ}\end{array}$ \\
\hline Volume & $\mathrm{c}=21.055(4) \AA \gamma=90^{\circ}$ \\
\hline Z, Calculated density & $3610.7(13) \AA^{3}$ \\
\hline Absorption coefficient & $4,1.465 \mathrm{mg} / \mathrm{m}^{3}$ \\
\hline$F_{(000)}$ & $0.114 \mathrm{~mm}^{-1}$ \\
\hline Crystal size (mm) & 1656 \\
\hline $\begin{array}{l}\text { Theta range for data } \\
\text { collection }\end{array}$ & $\begin{array}{l}0.32 \times 0.30 \times 0.25 \\
3.17-25.01^{\circ}\end{array}$ \\
\hline Limiting indices & $\begin{array}{l}-29 \Leftarrow \mathrm{h} \Leftarrow 29,-8 \Leftarrow \mathrm{k} \Leftarrow 8,-25 \Leftarrow 1 \\
\Leftarrow 25\end{array}$ \\
\hline Reflections collected/unique & $13632 / 3183\left[\mathrm{R}_{(\mathrm{int})}=0.1453\right]$ \\
\hline Completeness to theta $=25.01$ & $99.8 \%$ \\
\hline Absorption correction & Semi-empirical from equivalents \\
\hline Max. and min. transmission & 0.9720 and 0.9644 \\
\hline Refinement method & Full-matrix least-squares on $\mathrm{F}^{2}$ \\
\hline Data/restraints/parameters & 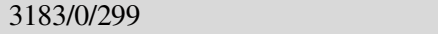 \\
\hline Goodness-of-fit on $\mathrm{F}^{2}$ & 1.047 \\
\hline Final $R$ indices $[I>2 \sigma(I)]$ & $\mathrm{R}_{1}=0.0709, \mathrm{wR}_{2}=0.1699$ \\
\hline $\mathrm{R}$ indices (all data) & $\mathrm{R}_{1}=0.1207, \mathrm{wR}_{2}=0.2047$ \\
\hline Largest diff. peak and hole & 0.498 and $-0.440 \mathrm{e} \AA^{-3}$ \\
\hline
\end{tabular}

The crystal packing is stabilized by $\mathrm{O}-\mathrm{H} \cdots \mathrm{O}$ and $\mathrm{O}-\mathrm{H} \cdots \mathrm{N}$ hydrogen bonding interaction. 


\begin{tabular}{lclc}
\hline \multicolumn{4}{c}{ TABLE-2 } \\
\multicolumn{4}{c}{ SELECT BOND LENGTHS [^] AND } \\
ANGLES [ ${ }^{\circ}$ ] FOR THE TITLE COMPLEX \\
\hline $\mathrm{N}(1)-\mathrm{C}(19)$ & $1.288(13)$ & $\mathrm{O}(3)-\mathrm{B}(1)-\mathrm{O}(6)$ & $109.3(3)$ \\
$\mathrm{N}(1)-\mathrm{C}(15)$ & $1.33(2)$ & $\mathrm{O}(3)-\mathrm{B}(1)-\mathrm{O}(1)$ & $112.8(3)$ \\
$\mathrm{O}(1)-\mathrm{C}(1)$ & $1.316(4)$ & $\mathrm{O}(6)-\mathrm{B}(1)-\mathrm{O}(1)$ & $110.3(3)$ \\
$\mathrm{O}(1)-\mathrm{B}(1)$ & $1.484(5)$ & $\mathrm{O}(3)-\mathrm{B}(1)-\mathrm{O}(4)$ & $106.3(3)$ \\
$\mathrm{O}(3)-\mathrm{B}(1)$ & $1.441(5)$ & $\mathrm{O}(6)-\mathrm{B}(1)-\mathrm{O}(4)$ & $112.0(3)$ \\
$\mathrm{O}(4)-\mathrm{C}(8)$ & $1.311(4)$ & $\mathrm{O}(1)-\mathrm{B}(1)-\mathrm{O}(4)$ & $106.0(3)$ \\
$\mathrm{O}(4)-\mathrm{B}(1)$ & $1.486(5)$ & $\mathrm{N}(1)-\mathrm{C}(15)-\mathrm{C}(16)$ & $121(2)$ \\
$\mathrm{O}(6)-\mathrm{C}(10)$ & $1.350(5)$ & $\mathrm{N}(1)-\mathrm{C}(15)-\mathrm{H}(15)$ & 119.7 \\
$\mathrm{O}(6)-\mathrm{B}(1)$ & $1.462(6)$ & $\mathrm{N}(1)-\mathrm{C}(19)-\mathrm{C}(18)$ & $121.3(10)$ \\
$\mathrm{C}(1)-\mathrm{O}(1)-\mathrm{B}(1)$ & $123.3(3)$ & $\mathrm{N}(1)-\mathrm{C}(19)-\mathrm{H}(19)$ & 119.3 \\
$\mathrm{C}(3)-\mathrm{O}(3)-\mathrm{B}(1)$ & $119.7(3)$ & $\mathrm{N}(1)-\mathrm{C}\left(15^{\prime}\right)-\mathrm{H}\left(15^{\prime}\right)$ & 119.2 \\
$\mathrm{C}(8)-\mathrm{O}(4)-\mathrm{B}(1)$ & $123.9(3)$ & $\mathrm{C}\left(18^{\prime}\right)-\mathrm{C}\left(19^{\prime}\right)-\mathrm{N}(1)$ & $117.8(6)$ \\
$\mathrm{C}(10)-\mathrm{O}(6)-\mathrm{B}(1)$ & $119.9(3)$ & $\mathrm{N}(1)-\mathrm{C}\left(19^{\prime}\right)-\mathrm{H}\left(19^{\prime}\right)$ & 121.1 \\
\hline
\end{tabular}

\section{ACKNOWLEDGEMENTS}

This study were supported by the Natural Science Foundation of Shandong Province (No. ZR2010BL025), Open Project of State Key Laboratory of Supramolecular Structure and Materials (No. sklssm201323)(Jilin University), State Key Laboratory of Inorganic Synthesis and Preparative Chemistry (No. 2011-13) (Jilin University) and the National Science Foundation of China (No. 21171132).

\section{REFERENCES}

1. X. Chen, H. Xue, X. Chang, L. Zhang, Y. Zhao, J. Zuo, H. Zang and W. Xiao, J. Alloys Comp., 425, 96 (2006).

2. L. Wu, X.L. Chen, Y. Zhang, Y.F. Kong, J.J. Xu and Y.P. Xu, J. Solid State Chem., 179, 1219 (2006).

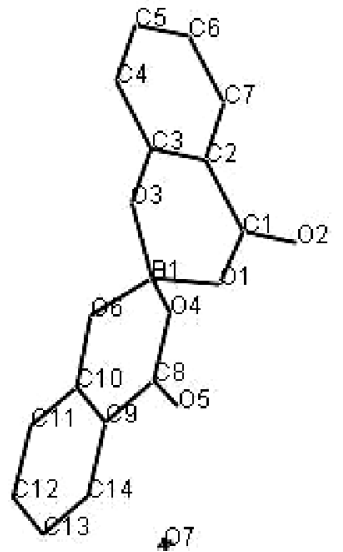

Fig. 1. Molecular structure of $\mathrm{C}_{38} \mathrm{H}_{34} \mathrm{~B}_{2} \mathrm{~N}_{2} \mathrm{O}_{16}$
3. H. Guler and B. Tekin, Inorg. Mater., 45, 538 (2009).

4. J. Zhang, J. Wang, X.Y. Huang and J.T. Chen, Z. Kristallogr. New Cryst. Struct., 220, 261 (2005).

5. Z.-H. Tang and C. Huang, Acta Cryst. E, 65, o171 (2009).

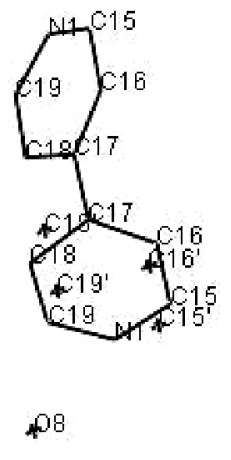

